\title{
MINIMAL SETS OF FAMILIES OF VECTOR FIELDS ON COMPACT SURFACES
}

\author{
JORGE HOUNIE
}

\section{Introduction}

Let $M$ be a compact connected smooth manifold of dimension two, and consider a subgroup $G$ of the group of diffeomorphisms of $M$. A set $\Omega \subset M$ is $G$-invariant if $g \Omega \subset \Omega$ for all $g$ in $G$. A set is said to be $G$-minimal if it is closed $G$-invariant nonempty, and contains no such proper subset. Let $D$ be a set of smooth vector fields on $M$, and consider the group $G_{D}$ generated by the one-parameter group whose infinitesimal generators are the elements of $D$. When $D$ contains exactly one vector field, a well-known theorem of Schwartz [5] shows that a $G_{D^{-}}$-minimal set is either a point, a homeomorph of $S^{1}$ or all of $M$ (in the last case $M$ must be homeomorphic to a torus $T^{2}$ ). The purpose of this paper is to extend this result to arbitrary families of vector fields.

Theorem 1. Let $M$ be a compact connected two-dimensional smooth manifold. Let $D$ be a set of smooth vector fields on $M$, and consider $a G_{D^{-m i n i m a l}}$ set $\Omega \subset M$. Then $\Omega$ must be one of the following:

(a) a point which is a common zero of the vector fields of $D$;

(b) $a G_{D^{-}}$orbit homeomorphic to $S^{1}$;

(c) all of $M$.

Proof. Let $m \in \Omega$, and denote by $\gamma(m)$ the $G_{D}$-orbit of $m$, i.e., the set of points of the form $g(m), g \in G_{D}$. By a theorem of Sussmann [7], $\gamma(m)$ is a smooth connected paracompact submanifold of $M$ (with a natural differentiable structure) of dimension $k, 0 \leqslant k \leqslant 2$. All vector fields in $D$ are tangent to $\gamma(m)$. If $k=0, \gamma(m)$ is a point and we have (a). If $k=2, \gamma(m)$ is open in $M$. Then $\overline{\gamma(m)} \backslash \gamma(m)$ is a closed invariant proper subset of $\Omega$, so $\overline{\gamma(m)}=\gamma(m)=$ $\Omega=M$. This gives (c). If $k=1, \gamma(m)$ is homeomorphic to $S^{1}$ or $\mathbf{R}$. In the first case we get (b). Assume that $\gamma(m)$ is homeomorphic to $\mathbf{R}$, and consider $\overline{\gamma(m)}=\Omega$. If the interior of $\Omega$ is nonempty, we conclude as before that $\Omega=M$. The theorem will be proved if we show that $\Omega$ cannot be nowhere dense when $\gamma(m)$ is homeomorphic to $\mathbf{R}$. Let us reason by contradiction, and assume that $\Omega$ is nowhere dense. 
Consider a vector field $X$ which belong to $D$ and does not vanish at $m$, and consider an imbedding $i:[-1,1] \rightarrow M$ such that

(a) $X$ is transversal to $i((-1,1))=I$,

(b) $i(-1)$ and $i(1)$ are not in $\Omega$,

(c) $i(0)=m$.

Given a point $p$ in $I \cap \Omega, \gamma(p)$ is homeomorphic to $\mathbf{R}$. In fact, we may choose a diffeomorphism $j: \mathbf{R} \rightarrow \gamma(p)$ so that $j(0)=p, j^{\prime}(0)=\lambda X(p), \lambda>0$. Since $\cap_{n} \overline{j[n, \infty)}$ is closed, invariant and nonempty, and is thus equal to $\Omega$, it follows that there is a least positive $s_{0}$ such that $j\left(s_{0}\right) \in I$ : " the first return to $I$ of the $G_{D}$-orbit through $p$ in the direction of $X$ ". It is easy to see that the vector $j^{\prime}(s), 0 \leqslant s \leqslant s_{0}$, can be extended to a vector field $Y$ in $M$, which is a finite linear combination with smooth coefficients of vector fields of $D$, that is, $Y$ belongs to the $C^{\infty}(M)$-module $D^{\prime}$ generated by $D$. So in a neighborhood of $p$ in $I$, the first return to $I$ of the $G_{D}$-orbit of a point in $\Omega \cap I$ is also the first return to $I$ through the orbit of $Y$. Since $\Omega \cap I$ is compact and nowhere dense in $I$, we may cover $\Omega \cap I$ with a finite number of disjoint open subsets of $I$, so that in each one of them the "first return" is performed through the orbit of a vector field of $D^{\prime}$. Thus the "first return function" can be extended to a smooth function $\tilde{f}$ in a neighborhood of $\Omega \cap I$ in $I$. The latter induces a smooth function $f=i^{-1} \tilde{f} i$, in a neighborhood $V$ of $i^{-1}(\Omega \cap I)=G, f: V \rightarrow$ $(-1,1)$.

In the same way, we obtain a smooth function $g: V \rightarrow(-1,1)$ induced by "the first return to $I$ of the $G_{D}$-oribt of $p$ in the direction of $-X$ ". Letting $W$ be open in $(-1,1)$ such that $G \subset W \subset \bar{W} \subseteq V$, we summarize the properties of $f$ and $g$ :

(1) $G=(-1,1) \backslash \cup_{i=1}^{\infty}\left(a_{i}, b_{i}\right), G$ is perfect,

(2) $H=\left\{a_{i}, b_{i}, i=1,2, \cdots\right\}, f(H) \subseteq H, g(H) \subseteq H$,

(3) $\left(a_{i}, b_{i}\right) \subset W$ implies $f\left(\left(a_{i}, b_{i}\right)\right)=\left(a_{j}, b_{j}\right), g\left(\left(a_{i}, b_{i}\right)\right)=\left(a_{k}, b_{k}\right)$ for some $j, k$,

(4) $f(G) \subset G, g(G) \subset G$.

(5) $0<L \leqslant\left|f^{\prime}(w)\right| \leqslant F, 0<L \leqslant\left|g^{\prime}(w)\right| \leqslant F$, for all $w \in W, 0<L<1<$ $F$,

(6) $\left|f^{\prime \prime}(w)\right| \leqslant M,\left|g^{\prime \prime}(w)\right| \leqslant M$, for all $w \in W$.

Consider the semigroup $S$ generated by $f$ and $g$, i.e., the functions $h: G \rightarrow G$ of the form $h=f^{n_{1}} \circ g^{m_{1}} \circ \ldots \circ f^{n_{j}} \circ g^{m_{j}}, n_{i}, m_{i} \in \mathbf{Z}^{+}$, where $f^{n}$ indicates composition $n$-times. We shall denote the $S$-orbit of $x$ by $[x], x \in G$. Then

(7) $i[x]=\gamma(i(x)) \cap \Omega, x \in G$,

(8) If $h \in S, a \in G$ and $h(a)=a$, there is a neighborhood $U$ of $a$ such that $h(b)=b$ for all $b$ in $U \cap G$. 
The last property is proved by observing that locally $f$ and $g$ are induced by the first return functions of certain vector fields $Y_{j}$, transversal to $I$. Therefore $h$ induces a piecewise differentiable path $\alpha$ made up to arcs of integral curves of the $Y_{j}$ 's. Since $h(a)=a$ and $\alpha \subseteq \gamma(i(a))$, each arc is traversed the same number of times in each direction. If $b \in G$ is sufficiently close to $a$, the path $\beta$ induced by $h$ starting at $i(b)$ will follow the arcs of integral curves of the same $Y_{j}$ 's used by $\alpha$ and in the same order. In particular, each arc will be traversed the same number of times in each direction. This implies that $h(b)=b$. Note that $U \cap G$ does not reduce to a point since $G$ is perfect.

To prove the theorem we need only show that properties (1) to (8) lead to a contradiction.

To each sequence of positive integers $\left(n_{1}, m_{1}, n_{2}, m_{2}, \cdots\right)$ we associate a sequence $F_{j}$ of functions of $S$ so that

$$
\begin{aligned}
& F_{0}=\text { identity, } \\
& F_{j}=f^{j-M_{k} \circ F_{M_{k}},} \quad M_{k}<j \leqslant N_{k+1}, \quad k=0,1,2, \cdots, \\
& F_{j}=g^{j-M_{k} \circ F_{N_{k}},} \quad N_{k}<j \leqslant M_{k}, \quad k=1,2, \cdots,
\end{aligned}
$$

where $N_{k}=n_{1}+m_{1}+\cdots+n_{k}, M_{k}=N_{k}+m_{k}, M_{0}=0$.

Lemma 1. There exist a complementary interval $(a, b), a, b \in G$, and $a$ sequence of positive integers $n_{1}, m_{1}, n_{2}, m_{2}, \cdots$ so that $F_{j}$ defined by (9) satisfies $F_{j}(a, b) \subset W, j=1,2, \cdots$, and $\left\{F_{j}(a), j=1,2, \cdots\right\}$ is dense in $G$.

Proof. Let $\mu=\operatorname{dist}(G,(-1,1) \backslash W), A=\left\{i \mid b_{i}-a_{i} \geqslant \mu\right\}$ and $B=\left\{a_{i}, b_{i}, i\right.$ $\in A\}$. The sets $A$ and $B$ are finite. By (7) we may identify $\left[a_{1}\right]$ with the integers $\mathbf{Z}$, where $k \in \mathbf{Z}$ corresponds to the $|k|$-th return to $I$ in the direction of $X$ or $-X$ according to the sign of $k$. Denote by $\bar{f}, \bar{g}, \bar{F}_{j}$ the functions induced by $f, g$, $F_{j}$ in this identification. Then $\bar{f}(k)=k \pm 1, \bar{g}(k)=k \pm 1$ and $|\bar{f}(k)-\bar{g}(k)|$ $=2$. Hence there is a sequence of positive integers $\left(n_{1}, m_{1}, \cdots\right)$ such that either $\bar{F}_{j}(0)=j$ or $\bar{F}_{j}(0)=-j, j=1,2, \cdots$, (according to the sign of $\bar{f}(0)$ ). It follows from (2) and the construction of $F_{j}$ that there exists $N$ such that $F_{k}\left(a_{1}\right) \notin B$ for $k \geqslant N$ and $F_{N}\left(a_{1}\right)=a_{i}$ or $b_{i}$ for some $i \notin A$. Hence $\left(a_{i}, b_{i}\right) \subset$ $W$, and it follows from (3) and the choice of $N$ that $\mid F_{j}\left(a_{i}\right)-F_{j}\left(b_{i}\right)<\mu$ for all $j=1,2, \cdots$. Then setting $(a, b)=\left(a_{i}, b_{i}\right), F_{j}((a, b)) \subseteq W$ for all $j=1,2, \cdots$.

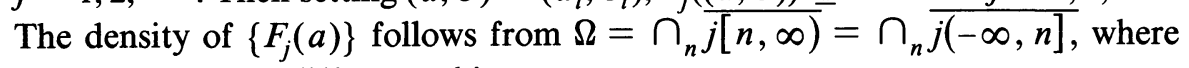
$j: \mathbf{R} \rightarrow \gamma(i(a))$ is a diffeomorphism.

Using Lemma 1, the mean value theorem and estimates (5) and (6) we may find, adapting the reasonings of $\left[5, \mathrm{p}\right.$. 456], a positive $\nu<\mu$ so that $\mid F_{j}(x)-$ $F_{j}(a) \mid<\mu$ for $|x-a|<\nu, j=1,2, \cdots$, and $F_{j}^{\prime}(x) \rightarrow 0$ uniformly for $|x-a|$ $\leqslant \nu, j \rightarrow \infty$, where $a$ is the left endpoint of the interval of Lemma 1 . 
Select $j$ such that

$$
\begin{aligned}
& \left|F_{j}^{\prime}(x)\right| \leqslant \frac{1}{2} \quad \text { if }|x-a| \leqslant \nu \\
& \left|F_{j}(a)-a\right| \leqslant \nu / 2
\end{aligned}
$$

It follows that $F_{j}:[a-\nu, a+\nu] \rightarrow[a-\nu, a+\nu]$ has a unique fixed point $p$ in $[a-\nu, a+\nu]$. Obtaining the fixed point by successive approximations starting at $a$ we see that $p \in G$. This contradicts (8).

Remarks. (1) Each one of the alternatives of Theorem 1 for a minimal set $\Omega$ actually occurs for suitable $D$. For instance, (c) is obtained if $D$ is such that to every point $p$ of $M$ there corresponds a pair of vectors of $D$ which are linearly independent at $p$. On the other hand, if $M=\Omega=\overline{\gamma(m)}$ but $\operatorname{dim} \gamma(m)=1, M$ must be homeomorphic to a torus $T^{2}$, since in this case any two vectors of $D$ are linearly dependent at every point of $M$, and $D$ defines a line field without singularities (see next section).

(2) When $D$ contains exactly one vector field, the functions $f$ and $g$ appearing in the proof of Theorem 1, satisfy $f=g^{-1}$, and the semigroup $S$ is a group, so proofs become simpler (see [5]).

(3) It is clear that "smooth" may be replaced by $C^{2}$ everywhere. A well-known example of Denjoy [1], showed that the theorem is false in the $C^{1}$ case.

\section{Line fields}

A smooth line field with singularities $\Lambda$ on a manifold $M$ is a smooth one-dimensional distribution defined on an open subset $V$ of $M$. The points of $M \backslash V$, where the distribution is not defined, are the singularities of $\Lambda$; if $V=M$ we say that $\Lambda$ is without singularities. By Frobenius theorem, the maximal integral curves of $\Lambda$ constitute a regular one-dimensional foliation of $V$. Thus we may consider an equivalence relation on $M$, whose equivalence classes are (i) the leaves of this foliation, and (ii) single points of $M \backslash V$. A subset of $M$ is $\Lambda$-invariant if it is a union of equivalence classes. A $\Lambda$-minimal set is a closed nonempty invariant set which contains no such proper subset. Two line fields with singularities $\Lambda_{1}, \Lambda_{2}$ defined on manifolds $M_{1}$ and $M_{2}$ respectively are equivalent if there exists a homeomorphism of $M_{1}$ onto $M_{2}$ which preserve the equivalence relations induced by $\Lambda_{1}$ and $\Lambda_{2}$. In particular, if $\Lambda_{1}$ and $\Lambda_{2}$ are equivalent, $M_{1}$ and $M_{2}$ are homeomorphic. 
A line field induced on $T^{2}=\mathbf{R}^{2} / \mathbf{Z}^{2}$ by a straight line with irrational angular coefficient will be referred to as "irrational line field on $T^{2}$ ".

Theorem 2. Let $M$ be a compact connected two-dimensional smooth manifold, and let $\Lambda$ be a smooth line field with singularities on $M$. Then a $\Lambda$-minimal set $\Omega$ must be one of the following:

(a) a singularity of $\Lambda$;

(b) a closed integral curve of $\Lambda$, homeomorphic to $S^{\mathbf{1}}$;

(c) all of $M$. In this case $\Lambda$ is equivalent to an irrational line field on $T^{2}$.

Proof. Let $V$ be the open subset of $M$ where $\Lambda$ is not singular, and consider a family of vector fields $D$ which vanish on $M \backslash V$ such that to every point $p$ of $V$, there are a neighborhood $U$ of $P$ and a vector field $X$ of $D$ which spans $\Lambda$ over $U$. It follows that $\Omega$ is $G_{D}$-minimal so (a), (b) or (c) or Theorem 1 must hold. If (c) holds, $\Lambda$ has no singularities. This implies (see for instance [3, p. 275]) that the Euler characteristic of $M$ is zero, so $M$ is homeomorphic to a torus $T^{2}$ or a Klein bottle $K^{2}$. In the latter case, every regular one-dimensional foliation of $M$ has a closed leaf (Kneser [2, p. 153]), so $\Omega$ cannot be all of $M$. Then $M$ must be homeomorphic to $T^{2}$. Consiider a smooth closed curve $\Gamma$ everywhere transversal to $\Lambda$, and consider a vector $X \neq 0$ on $\Gamma$ which spans $\Lambda$ over $\Gamma$. Let $f(x)$ be the first return to $\Gamma$ of the leaf through $x$ in the direction of $X$. Suppose that for a certain $x \in \Gamma$ the arc of integral curve of $\Lambda$ which joins $x$ to $f(x)$ enters $\Gamma$ in the direction of $-X$. Then the same will happen for all $x \in \Gamma$ since the set of those points is open and closed in $\Gamma$. This implies that $f$ reverses the orientation of $\Gamma$ and has a fixed point, which is impossible. Thus the arcs leaving $\Gamma$ in the direction of $X$, also enter $\Gamma$ in the direction of $X$. This induces a coherent orientation on the leaves of $\Lambda$, and $\Lambda$ may be spanned by a single vector field $X_{1}$ which extends $X$. The "first return to $\Gamma$ " function induced by $X_{1}$ must have an irrational rotation number. Therefore $\Lambda$ is equivalent to an irrational line field on $T^{2}$ [6, Chap. III].

Remark. Related results concerning line fields spanned by a single vector field were studied in [4, p. 210]. When the set $V$ where $\Lambda$ is regular is simply connected, $\Lambda$ is spanned by a single vector field. However, this is not true in general, as simple examples show.

\section{References}

[1] A. Denjoy, Sur les courbes définies par des équations differentielles à la surface du tore, J. Math. Pures Appl. 11 (1932) 333-375.

[2] H. Kneser, Regulare Kurvenscharen auf den Ringflächen, Math. Ann. 91 (1924) 135-154.

[3] J. Little, On singularities of submanifolds of higher dimensional Euclidean spaces, Ann. Mat. Pura Appl. 83 (1969) 261-335. 
[4] R. Sacksteder \& A. Schwartz, Limit sets of foliations, Ann. Inst. Fourier (Grenoble) 15 (1965) 201-213.

[5] A. Schwartz, A generalization of the Poincaré-Bendixon theorem to closed two-dimensional manifolds, Amer. J. Math. 85 (1963) 453-458.

[6] S. Sternberg, Celestial mechanics, Part II, Benjamin, New York, 1969.

[7] H. Sussmann, Orbits of families of vector fields and integrability of distributions, Trans. Amer. Math. Soc. 180 (1973) 171-188.

Universidade Federal de Pernambuco RECIFE, BRASIL 